\title{
Validación y análisis de eficiencia en la producción de agua mediante la implementación de celdas Peltier
}

\section{Validation and analysis of efficiency in water production through the implementation of Peltier cells}

CHÁVEZ, José Manuel †**, ESPEJEL-BLANCO, Daniel Fernando, HERNÁNDEZ-AGUIRRE, Fredy Alberto y RASCÓN-BARCELÓ, Luis Eduardo

Tecnológico Nacional de México / Instituto Tecnológico De Hermosillo, México.

ID 1 ${ }^{\text {er }}$ Autor: José Manuel, Chávez / ORC ID: 0000-0001-9708-5925, CVU CONACYT ID: 806766

ID $1^{\text {er }}$ Coautor: Daniel Fernando, Espejel-Blanco / ORC ID: 0000-0002-9692-013X, CVU CONACYT ID: 304845

ID $2^{\text {do }}$ Coautor: Fredy Alberto, Hernández-Aguirre / ORC ID: 0000-0001-9208-5299, CVU CONACYT ID: 611800

ID $3^{\text {er }}$ Coautor: Luis Eduardo, Rascón-Barceló / ORC ID: 0000-0001-6051-9777, CVU CONACYT ID: 929775

DOI: $10.35429 / J U S D .2020 .19 .6 .22 .30$

Recibido: 15 de Julio, 2020; Aceptado 30 de Diciembre, 2020

\section{Resumen}

En la actualidad, las temporadas de sequía son más prolongadas a consecuencia del calentamiento global, lo cual ha generado escases y racionamiento de agua, dichas condiciones se convierten en el principal problema en los poblados alejados a las zonas urbanas y con poca infraestructura. México cuanta con 653 acuíferos en todo su territorio, 106 están sobreexplotados debido a actividades agrícolas, mineras, industriales y urbanas, lo que proyecta al país un posible agotamiento y contaminación de su líquido vital. Por tal motivo, con el fin de contribuir con una solución a la problemática de la escases de agua, al aplicar un sistema de control a la tecnología que utilizan las celdas de Peltier se genera un flujo moderado de agua, ya que condensa las partículas de agua suspendidas en el aire. De esta manera, se desarrolló un prototipo en el cual se implementa un arreglo de celdas de Peltier para obtener la mayor cantidad agua posible bajo factores ambientales, como humedad y temperatura, así como un análisis de eficiencia, costo y viabilidad con un sistema comercial generador de agua con capacidades similares.

Energías renovables, Condensación de agua, Celdas de Peltier

\begin{abstract}
At present, drought seasons are longer as a result of global warming, which has generated scarcity and rationing of water, these conditions become the main problem in towns far from urban areas and with little infrastructure. Mexico, with 653 aquifers throughout its territory, 106 are overexploited due to agricultural, mining, industrial and urban activities, which projects a possible depletion and contamination of its vital liquid to the country. For this reason, in order to contribute to a solution to the problem of water scarcity, when applying a control system to the technology used by Peltier cells, a moderate flow of water is generated, since it condenses the particles of water suspended in the air. In this way, a prototype was developed in which a Peltier cell arrangement is implemented to obtain as much water as possible under environmental factors, such as humidity and temperature, as well as an analysis of efficiency, cost and viability with a commercial generator system of water with similar capacities.
\end{abstract}

Renewable energies, Water condensation, Peltier Cells

Citación: CHÁVEZ, José Manuel, ESPEJEL-BLANCO, Daniel Fernando, HERNÁNDEZ-AGUIRRE, Fredy Alberto y RASCÓN-BARCELÓ, Luis Eduardo. Validación y análisis de eficiencia en la producción de agua mediante la implementación de celdas Peltier. Revista del Desarrollo Urbano y Sustentable. 2020. 6-19:22-30.

\footnotetext{
*Correspondencia al autor (Correo electrónico: jmgarcia@uaz.edu.mx)

$\dagger$ Investigador contribuyendo como primer autor
} 


\section{Introducción}

En la actualidad, el hablar sobre el cambio climático es referirse al principal problema que nuestro planeta enfrenta y el cual, no solo afecta a nuestros distintos ecosistemas, sino también a la sociedad.

Las temperaturas del planeta son aptas para la vida gracias a un proceso natural llamado "Efecto Invernadero"; cuando la radiación solar llega a nuestra atmosfera, parte de ella es reflejada hacia el espacio y otra parte es absorbida por la Tierra, obteniendo la temperatura adecuada para la vida en ella. Por otra parte, existen los llamados "Gases de Efecto Invernadero", los cuales evitan que la radiación solar salga hacia el espacio y se quede en nuestra atmosfera, ocasionando un incremento en las temperaturas.

México no es la excepción, a consecuencia del calentamiento global el país experimenta distintos problemas climáticos, los cuales nunca se habían registrado. El incremento de temperatura cada vez es más prolongado en épocas de sequía, donde el suelo es árido, se ha generado escases y racionamiento de agua, dichas condiciones se convierten en la mayor afectación en los pueblos alejados a las zonas urbanas y con poca infraestructura. . El agua con el que se cuenta, muchas veces por condiciones naturales, y otras por la intervención del ser humano, llega a sufrir daños irreversibles. México cuenta con 653 acuíferos en todo su territorio, 106 están sobreexplotados debido a actividades agrícolas, mineras, industriales y urbanas, lo que proyecta al país un posible agotamiento y contaminación de su líquido vital (Comisión Nacional del Agua, 2018).

Al tratarse de zonas donde el clima es árido, el acceso a fuentes ilimitadas de este líquido vital es imposible, afectando al desarrollo de empresas y comunidades. Se han realizado múltiples investigaciones sobre los métodos alternativos para la obtención de agua, logrando resultados no viables. Dado a los altos costos de transportación de agua potable entre distintas localidades y a la sobreexplotación de los mantos acuíferos pareciera que nuestro problema no tendrá fin.
Ante el evidente problema que nuestro planeta enfrenta a consecuencia del cambio climático y el racionamiento del agua, como ingenieros y futuros investigadores es nuestra obligación desarrollar y aplicar nuevas tecnologías para aprovechar los recursos del planeta, como es el caso de la implementación de sistemas fotovoltaicos.

Por otra parte, el aprovechamiento de la energía solar para generar electricidad a través de la tecnología fotovoltaica se ha convertido en una opción cada vez más asequible para los 1400 millones de personas en el mundo que viven aún sin energía eléctrica (Rodriguez \& Espinoza, 2017). Dependiendo de las necesidades humanas, el sistema fotovoltaico puede variar en tipo, capacidad o especificaciones. Así, los tipos de sistemas fotovoltaicos se dividen en dos principales: los autónomos o aislados y los interactivos con la red eléctrica o interconectados.

El funcionamiento de un sistema fotovoltaico es posible gracias al a los paneles solares donde gracias al efecto fotoeléctrico, la energía solar se convierte en energía eléctrica de corriente directa, el cual puede ser convertido a corriente altera al utilizar el inversor, esto dependerá de los dispositivos electrónicos que se deseen alimentar con el sistema fotovoltaico (Style, 2012). Posteriormente, dependiendo del tipo de sistema fotovoltaico: autónomo o interconectado, en el caso de sistemas fotovoltaicos autónomos es necesario utilizar controladores de cargas que regulan el uso de la energía y un banco de baterías que permita el almacenamiento de la energía. Por otra parte, el uso de centros de carga es fundamental en sistemas fotovoltaicos interconectados, ya que es el punto de conexión o de distribución de energía fotovoltaica generada para que el medidor bidireccional registre la energía que se regresa a la red eléctrica de CFE (Rodriguez \& Espinoza, 2017).

El objetivo de este proyecto es la implementación de un prototipo generador de agua utilizando Celdas Peltier, basadas en el principio termoeléctrico Peltier, para condensar el vapor de agua contenido en el aire. 
Los dispositivos Peltier se usan principalmente para refrigeración, en estado sólido transfiere calor de un lado del dispositivo a otro oponiéndose al gradiente de temperatura, consumiendo para ello energía eléctrica. Las compañías de automóviles alemanas Volkswagen y BMW han desarrollado generadores termoeléctricos (GTE) que recuperan el gasto de calor de una máquina de combustión. Volkswagen afirma $600 \mathrm{~W}$ de salida del GTE en condición de conducción en autopista. La electricidad producida por el GTE es cerca del $30 \%$ de la electricidad requerido por el auto, obteniendo una carga mecánica reducida (alternador) y una reducción en el consumo de combustible de más del $5 \%$. (Díaz T., 2015)

La implementación y pruebas del prototipo fueron realizadas en el municipio de Hermosillo, Sonora. Los datos de estudio obtenidos fueron tomados de WeatherSpark (Weather Spark, 2019), el cual es un sitio web que se encarga de reunir información climatológica a nivel mundial. Al realizar un análisis de los datos climatológicos recabados los últimos 5 años (2013-2018) determinamos que los datos presentan aproximaciones entre sí en cada uno de los años analizados. Además, al tratarse de zonas donde la distribución de energía eléctrica es inaccesible, el uso de fuentes de energía de tipo fotovoltaico servirá para alimentar al sistema generador de agua de una manera autosustentable aprovechando la radiación solar.

\section{Desarrollo}

Una forma de obtener un flujo constante de agua a partir de la humedad del aire se logra a través de la implementación de Celdas Peltier para condensar el vapor de agua contenido en el ambiente. El punto de rocío o temperatura de rocío es la más alta temperatura a la que empieza a condensarse el vapor de agua contenido en el aire, produciendo rocío, neblina, cualquier tipo de nube o, en caso de que la temperatura sea lo suficientemente baja, escarcha (MetAs \& Metrologos Asociados, 2007). Con este sistema es posible obtener la mayor cantidad de agua posible bajo factores ambientales, como humedad y temperatura, en relación con la manipulación y control de las Celdas de Peltier.
El prototipo está complementado con sensores para la medición de temperatura y humedad, las cuales determinaran las condiciones de activación del prototipo utilizando un control ON/OFF mediante la plataforma de Arduino. El cual es una plataforma de creación de electrónica de código abierto, la cual está basada en hardware y software libre, flexible y fácil de utilizar para los creadores y desarrolladores, la cual crear diferentes tipos de microordenadores de una sola placa a los que la comunidad de creadores puede darles diferentes tipos de uso (Electronics, s.f.).

La estructura principal del prototipo consiste básicamente en cuatro etapas, como se puede observar en la Figura 1: Activación del Dispositivo, Etapa de Muestreo, Etapa de Potencia y Conexión al Sistema de Alimentación Fotovoltaico, siendo las dos primeras interconectadas a través de una arquitectura desarrollada a partir de la plataforma Arduino.

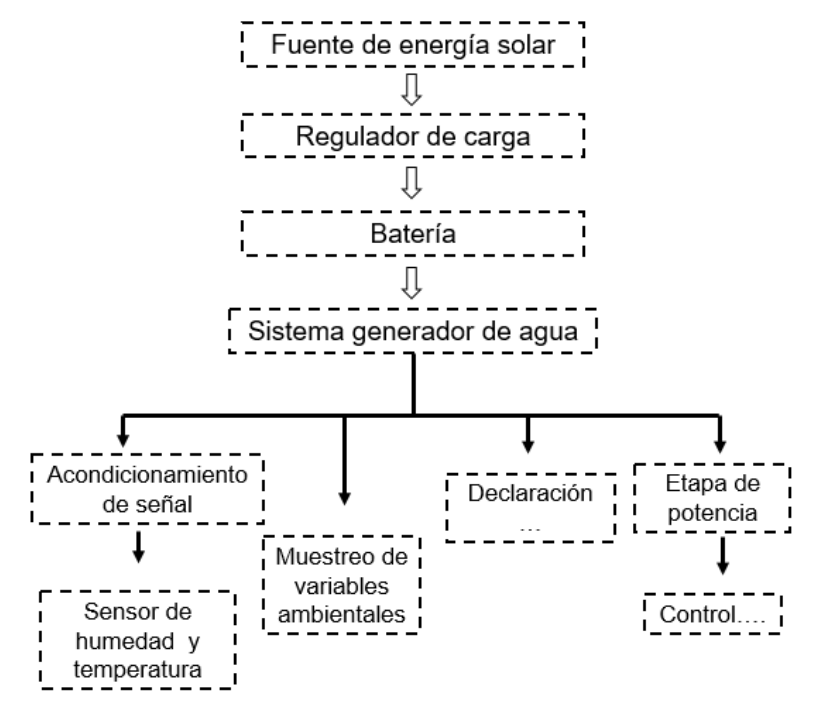

Figura 1 Descripción de Bloques de Funcionamiento

El apartado Activación del Dispositivo muestra el modo de activación del sistema y la interfaz gráfica donde se almacenan los datos de las variables a medir.

En el punto Etapa de Muestreo se lleva a cabo el muestreo y el procesamiento de variables que intervienen en el prototipo, las cuales son utilizados para en el control y activación del sistema bajo las condiciones ideales para su funcionamiento. 
En el punto Etapa de Potencia se desarrolla el circuito de potencia para la activación de las Celdas Peltier. Por último, la conexión del prototipo a su fuente de alimentación, fotovoltaica, se presenta en el apartado Conexión al Sistema de Alimentación Fotovoltaico.

\section{Activación del dispositivo}

Al encender por primera vez el dispositivo, Arduino Mega iniciara en modo "Desconectado" en el cual el Sistema Generador se encontrara apagado, al igual, los sensores no estarán en operación. Sin contar el modo "Desconectado", el sistema tiene otro método de operación:

- Modo PLX-DAQ: Parallax Data Acquisition tool PLX-DAQ es un complemento para MS Excel 2000 y 2003, que te permite capturar hasta 26 canales de datos de un Arduino conectado a una puerto serie (COM1 15), con velocidad de hasta 128 Kbps. Puedes adjuntar fecha y hora del evento (hh:mm:ss) desde el sistema o segundos desde el inicio, Figura 2.

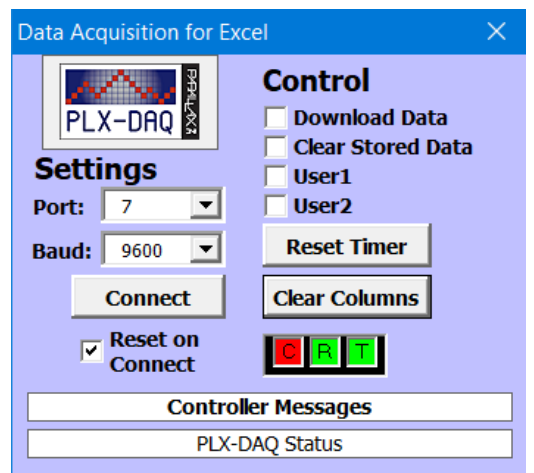

Figura 2 Software PLX-DAQ para la Captura y Almacenamiento de Datos de Variables Ambientales

Para ingresar a este modo debe conectarse el dispositivo Arduino Mega al software PLX-DAQ y ejecutar el programa, el cual, mostrara los datos guardados de las variables ambientales en tiempo real, complemento de Excel. Cabe destacar que dicho software es de solo muestreo, las condiciones de activación y los datos de muestreo los realiza Arduino Mega.

\section{Etapa de muestreo}

Para la adquisición de datos se utilizó la plataforma de programación Arduino, en conjunto con sensores de medición de humedad y temperatura. A través de la plataforma de Arduino y la tarjeta de adquisición Arduino Mega 2560, es posible la interacción con distintos tipos de sensores. Se implementó el sistema para realizar el muestreo de las variables ambientales. Las condiciones de activación, Figura 3, fueron definidas en base a la humedad en el ambiente, se estableció en cada condición el encendido de las Celdas Peltier y resistencias generadoras de calor.

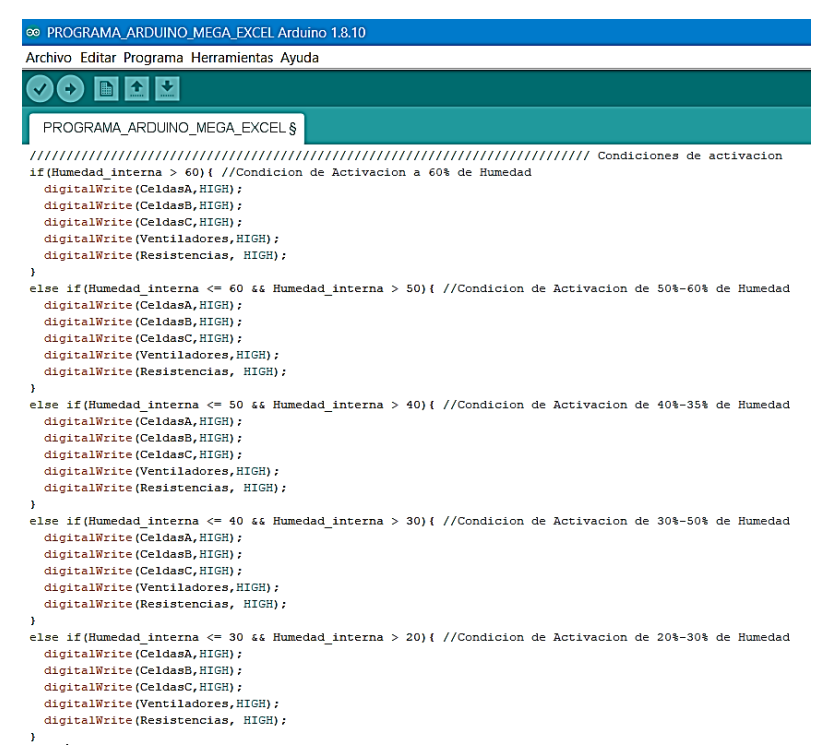

Figura 3 Programa en Arduino para controlar Arduino Mega y las Condiciones de Activación de las Celdas Peltier

Al detectar niveles óptimos de humedad y temperatura, anteriormente definidos en la programación, se enviará una señal de activación a la etapa de potencia, encendiendo las Celdas Peltier.

Al integrar en el prototipo un sistema interno de calefacción, Figura 4, utilizando resistencias de calor, es posible controlar y modificar las variables ambientales contenidas en el aire, lo cual beneficiara la producción del punto de rocío. 


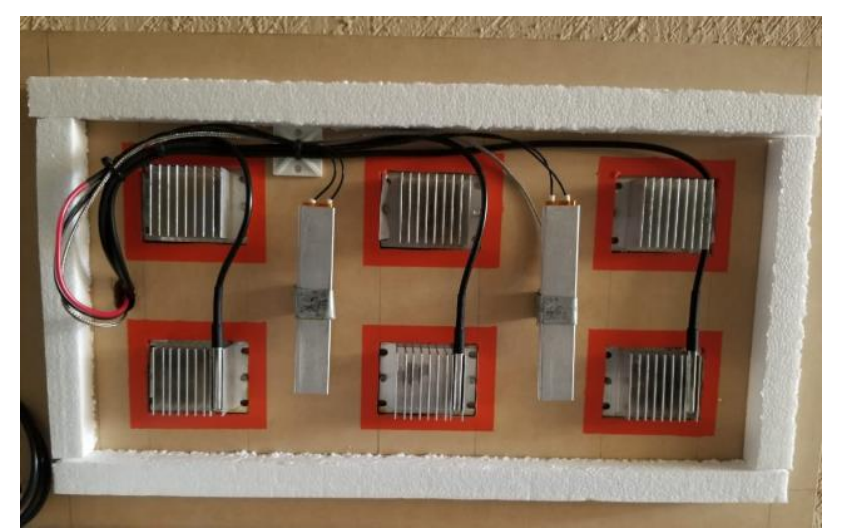

Figura 4 Montaje de resistencias calefactoras

El tiempo de encendido de las resistencias calefactoras está definido en la programación, como se muestra en la Figura 5, y relacionada directamente con las condiciones de activación de las Celdas en base a la humedad en el ambiente. Es decir, mientras mayor sea el porcentaje de humedad el tiempo de encendido de las resistencias calefactoras será menor. Este tipo de resistencias emiten calor cuando se le aplica una corriente eléctrica, entonces una parte de la energía cinética contenida en los electrones del material se transforma en calor.

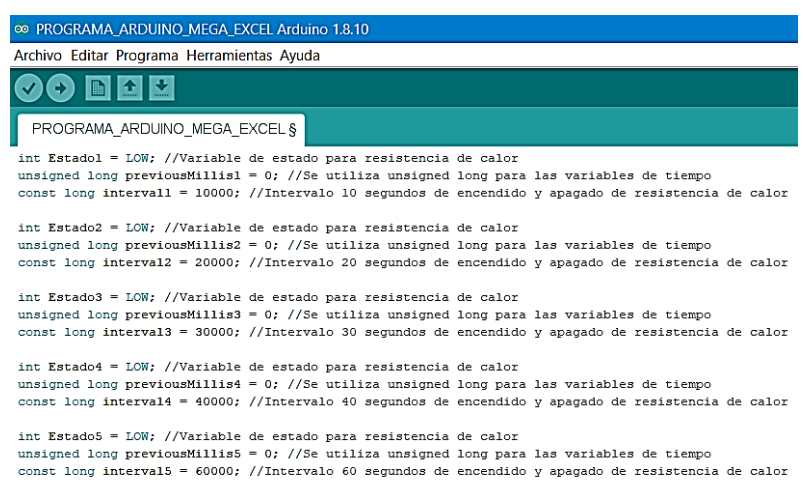

Figura 5 Programa en Arduino para controlar Arduino Mega y las condiciones de activación de las resistencias de calor por periodos de tiempo

\section{Etapa de potencia}

Al utilizar la tarjeta Mega 2560 y su plataforma Arduino, el sistema es puesto en funcionamiento en base a las variables medidas, humedad relativa y temperatura, con la finalidad de entrar en operación cuando las variables mencionadas sean óptimas para la mayor obtención de rocío. Si los factores ambientales son ideales, la tarjeta Mega 2560 envía una señal por medio de su salida digital al sistema de potencia, Figura 6, que a su vez activará a la Celda Peltier o Resistencia de Calor.

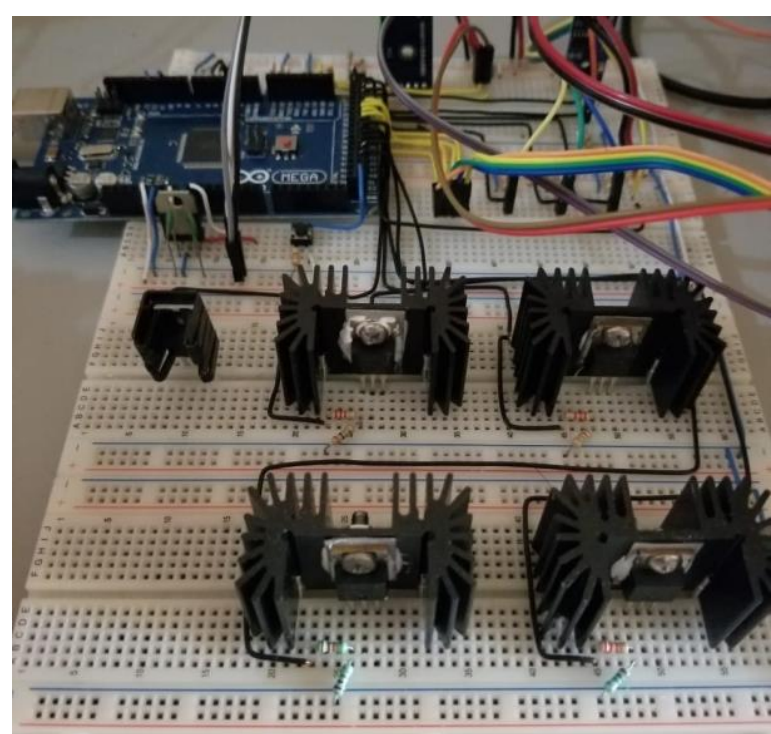

Figura 6 Interfaz Arduino - Etapa de Potencia

La etapa de potencia está conformada por dispositivos MOSFET, los cuales son utilizados para la conmutación y amplificación de señales digitales. Existen muchos modelos de MOSFET disponibles, pero no todos son recomendados para usar directamente con un procesador como Arduino por la limitación de tensión e intensidad en sus salidas. Así, la serie de transistores lógicos, por ejemplo, IRL540, se saturan sin problemas a $5 \mathrm{~V}$ proporcionando una Id de 28A, suficiente para soportar la corriente necesaria por las Celdas Peltier para su funcionamiento.

\section{Conexión al sistema de alimentación fotovoltaico}

Para realizar la instalación del sistema fotovoltaico es necesario comprender el esquema del sistema, Figura 7, pues éste nos orientará en el proceso de instalación de forma eficiente y adecuada. Al tratarse de zonas donde la distribución de energía eléctrica es inaccesible, el uso de un sistema aislado es la mejor opción para suministrar energía al generador de agua, esto se hace a través de un banco de baterías que almacene electricidad y la mantiene disponible para cuando sean necesario. 


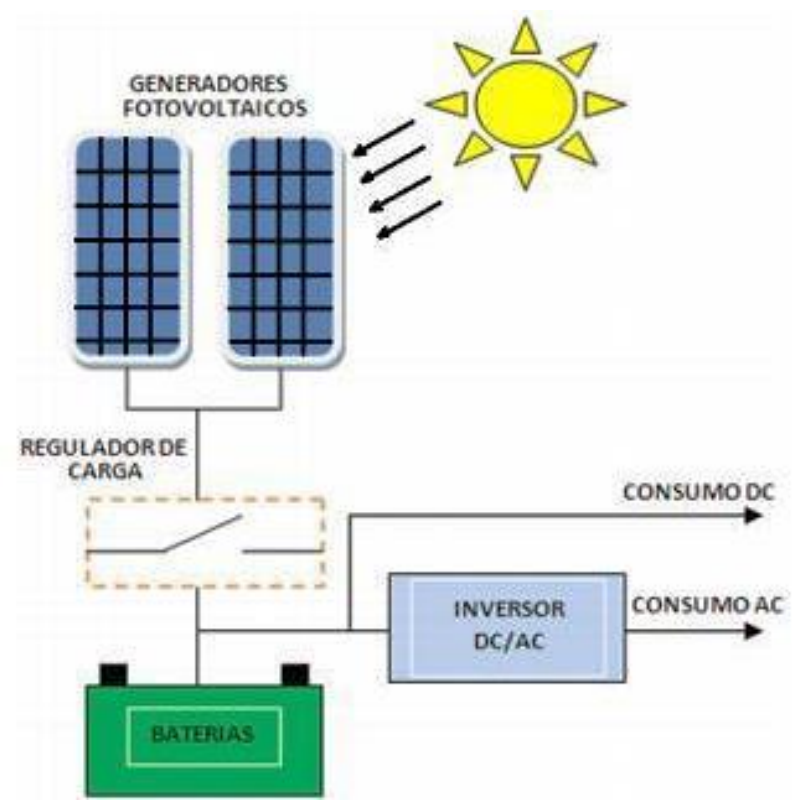

Figura 7 Esquema de instalación del sistema fotovoltaico

La electricidad en corriente continua es la que proviene de los módulos fotovoltaico y que se almacena en las baterías. Si el prototipo es puesto en operación durante el día utilizará directamente la energía generada por los módulos fotovoltaicos, contrario a esto, en ausencia de luz solar el sistema se alimentará de los acumuladores, que previamente almacenaron energía eléctrica durante el día.

\section{Resultados}

Los resultados obtenidos demuestran el desempeño de las tecnologías para resolver las necesidades básicas de la sociedad. Al diseñar nuevos métodos o sistemas para la obtención de agua, podemos aprovechar los distintos ecosistemas y estaciones del año para generar la máxima cantidad posible, de una manera amigable para el medio ambiente y a un bajo costo. La cantidad de agua generada será proporcional al número de Celdas Peltier utilizadas, la humedad presente en el ambiente y el tiempo de operación del sistema.

\section{Pruebas preliminares de prototipos}

Para la elección de la estructura del prototipo se procedió a realizar pruebas experimentales con diferentes configuraciones de celdas Peltier, se utilizó un Kit-Refrigeración (modelo TEC112706), dichas pruebas se llevaron a cabo como base para comprobar la viabilidad y la configuración del sistema de condensado.
Después de probar varios arreglos de celdas durante la etapa de experimentación, en base a los resultados obtenidos, se decidió redimensionar el prototipo para la utilización de 6 celdas Peltier distribuidas como se muestra en la Figura 8.

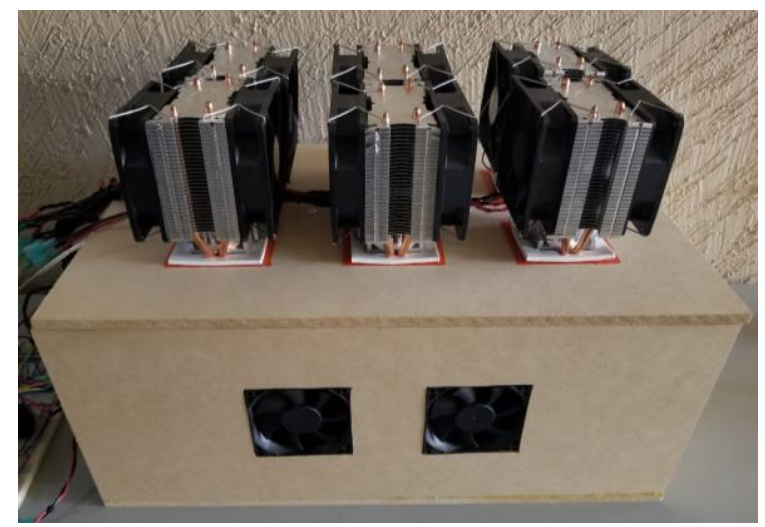

Figura 8 Prototipo \#3

Una vez realizadas las pruebas preliminares se eligió el prototipo \#3 para su implementación final. Cabe recordar, la función de la estructura es mantener y/o disminuir la temperatura a la cual se encuentra el vapor de agua que ingresa a través de él. Al aumentar el volumen de la estructura, las partículas (átomos o moléculas) de vapor de agua tardan más en llegar a las paredes del recipiente y por lo tanto chocan menos veces por unidad de tiempo contra ellas. Esto significa que la presión será menor ya que ésta representa la frecuencia de choques del gas contra las paredes. La presión del gas es directamente proporcional a su temperatura, esto quiere decir, si el volumen de la estructura es mayor la presión disminuirá por lo tanto su temperatura disminuirá proporcionalmente lo cual beneficiará la condensación del vapor de agua, como se puede observar en la Tabla 1.

\begin{tabular}{|r|r|r|r|}
\hline Prototipo & $\begin{array}{c}\text { Número } \\
\text { de celdas }\end{array}$ & $\begin{array}{c}\text { Dimensiones } \\
\text { (cm) }\end{array}$ & $\begin{array}{c}\text { Agua } \\
\text { obtenida } \\
\text { (mL) }\end{array}$ \\
\hline$\# 1$ & 1 & $15 \times 15 \times 15$ & 15 \\
\hline$\# 2$ & 4 & $40 \times 20 \times 28$ & 85 \\
\hline$\# 3$ & 6 & $40 \times 25 \times 28$ & 200 \\
\hline
\end{tabular}

Tabla 1 Eficiencia en la producción de agua en relación con número de celdas/dimensión de estructura

\section{Metodología general}

En todos los casos, se comparó la relación entre la potencia consumida por la cantidad de agua obtenida. 
Se tomaron 6120 muestras utilizando el prototipo \#3 en tres casos distintos y el software PLX-DAQ para el almacenamiento de datos, en el primer caso, utilizando solo una Celda Peltier, el segundo caso cuatro Celdas Peltier y por último, seis Celdas Peltier. Cada una de las pruebas bajo condiciones climáticas promedio: Humedad Ambiente 45\%, Temperatura Ambiente $25^{\circ} \mathrm{C}$, Tiempo de Trabajo 12 horas.

\begin{tabular}{|l|r|r|r|}
\hline & \multicolumn{1}{c}{$\begin{array}{c}\text { Potencia } \\
\text { consumida } \\
\text { (Wh) }\end{array}$} & \multicolumn{1}{c|}{$\begin{array}{c}\text { Prod. de } \\
\text { agua (mL) }\end{array}$} & $\begin{array}{c}\text { Relación } \\
\text { (W/mL) }\end{array}$ \\
\hline Caso \#1 & 45.82 & 25 & 1.83 \\
\hline Caso \#2 & 183.36 & 128 & 1.43 \\
\hline Caso \#3 & 275.04 & 200 & 1.37 \\
\hline
\end{tabular}

Tabla 2 Gasto energético en la producción de agua en relación con número de celdas/prototipo \#3

Los resultados obtenidos, Tabla 2, muestra la relación entre la potencia consumida y los distintos casos en los cuales se utilizaron las Celdas Peltier para producir cierta cantidad de agua utilizando el dispositivo \#3 bajo las condiciones mencionadas anteriormente.

\section{Análisis Comparativo}

De forma previa a la comprobación de la hipótesis planteada, se llevó a cabo un análisis de un sistema comercial generador de agua, con el cual el sistema fue puesto a comparación en eficiencia, costo y viabilidad. Con el fin de analizar la relación de los dos sistemas se consideró su funcionamiento bajo los mismos parámetros y el redimensionamiento del prototipo \#3, dando como resultado la Tabla 3.

\begin{tabular}{|l|r|r|}
\hline \multicolumn{2}{|c|}{$\begin{array}{c}\text { Source } \\
\text { Hydropanels }\end{array}$} & Prototipo Generador \\
\hline $\begin{array}{l}\text { Costos (Instalación, } \\
\text { materiales, construcción) }\end{array}$ & $\$ 76,120$ & $1,000 \mathrm{~mL}$ \\
\hline $\begin{array}{l}\text { Producción de Agua } \\
\text { (mL) }\end{array}$ & $1,000 \mathrm{~mL}$ & Sí \\
\hline $\begin{array}{l}\text { Sistema de Control } \\
\text { (Eficiencia) }\end{array}$ & & $24 \mathrm{Hr}$ \\
\hline $\begin{array}{l}\text { Tiempo de Activación } \\
\text { (Por día o 24 horas) }\end{array}$ & $12 \mathrm{Hr}$ & 20 Años \\
\hline $\begin{array}{l}\text { Tiempo de Vida del } \\
\text { Sistema (Años) }\end{array}$ & 10 Años & Fotovoltaica/Autónomo \\
\hline $\begin{array}{l}\text { Alimentación } \\
\text { (Fotovoltaica/Autónomo) }\end{array}$ & Fotovoltaica & Fon \\
\hline $\begin{array}{l}\text { Control sobre Variables } \\
\text { Ambientales }\end{array}$ & & Sí \\
\hline $\begin{array}{l}\text { Relación Potencia } \\
\text { Consumida/Agua } \\
\text { Generada }\end{array}$ & $4.285 \mathrm{~W} / \mathrm{mL}$ & \\
\hline Costo lt/diario (M.N.) & $\$ 20.85$ & \\
\hline
\end{tabular}

Tabla 3 Desempeño SOURCE Hydropanels vs Prototipo
Como puede observarse en la Tabla 2 y Tabla 3, gracias a la validación de los casos presentados, demuestra la obtención de la mayor cantidad de agua mediante el prototipo desarrollado. En el caso número tres, donde se utilizó la mayor cantidad de Celdas Peltier, se obtuvo los mejores resultados bajo condiciones climáticas similares, si el volumen de la estructura es mayor, la presión disminuirá por lo tanto su temperatura disminuirá proporcionalmente lo cual beneficiará la condensación del vapor de agua.

El prototipo propuesto para la generación de agua es una excelente opción en zonas donde no exista su acceso, ya que no requiere un alto costo de implementación. Los resultados obtenidos con las pruebas realizadas, nos brinda las bases necesarias para implementar un sistema generador y adaptarlo en sitios con alto porcentaje de humedad. Ya que el sistema de control funciona bajo los mismos criterios, solo es necesario dimensionar el prototipo dependiendo de la cantidad de agua que generara y sus aplicaciones. Por otra parte, puede ser implementado en zonas donde no exista suministro constante de energía eléctrica, gracias a su fuente de alimentación autosustentable.

\section{Agradecimiento}

Agradecimiento especial al Tecnológico Nacional de México/Instituto Tecnológico de Hermosillo, por proporcionar los fondos para que este proyecto se llevara a cabo.

\section{Conclusiones}

En este proyecto se desarrolló un sistema prototipo capaz de generar agua a partir de la humedad que se encuentra en el ambiente. Para esto se investigó y se implementaron métodos para la utilización de Celdas Peltier, con la finalidad de condensar el vapor de agua contenido en el ambiente, así como circuitos de potencia para su aplicación y el desarrollo de software capaz de optimizar resultados. Posteriormente se seleccionó la estructura, en conjunto con las Celdas Peltier, se obtenía la mayor cantidad de agua y determinar en qué condiciones climatológicas es recomendable implementarlo. 
El resultado más importante de este proyecto es que se demuestra que es posible obtener agua a partir de la humedad que se encuentra en el ambiente, sin importar que los niveles de humedad y temperatura sean mínimos.

\section{Trabajos futuros y recomendaciones}

El prototipo propuesto sugiere algunas pruebas $\mathrm{y}$ modificaciones tanto a nivel hardware como software. Se propone la utilización de la plataforma WIFI, en reemplazo a la plataforma Arduino, para el monitoreo y control del prototipo generador. Con lo cual se tendrá un mejor manejo de datos a distancia interconectados a la red local y a su vez, una disminución de dimensionamiento de circuitería.

Se recomienda la construcción de una estructura la cual esté sellada herméticamente y cuente con un alto nivel de limpieza, es decir, al generar agua se deberá depositar en un lugar fresco y libre de microorganismos que puedan afectar su calidad.

Por otra parte, el agua generada por el prototipo va alimentándose de materias extrañas que se encuentran a su paso. Así, el agua en estado puro, en muchas ocasiones no es apta para el consumo humano, por el bien de su salud, el agua pasa por un proceso de depuración y sanación, que vendrá determinada según su grado de contaminación, que puede averiguarse mediante un llamado análisis del agua.

\section{Referencias}

Comisión Nacional del Agua. (10 de Abril de 2018). "Situación de los Recursos Hídricos". Obtenido de gob.mx: https://www.gob.mx/conagua/acciones-yprogramas/situacion-de-los-recursos-hidricos

Electronics, M. (s.f.). Arduino.cl. Recuperado el 5 de Abril de 2019, de http://arduino.cl/que-esarduino/

Eole Water Company. (s.f.). Recuperado el 10 de Noviembre de 2018, de Eole Water Company: http://www.eolewater.com/
Díaz T., D. (2015). Efecto termoeléctrico. Descripción y aplicación. Universidad Simón Bolivar, Miranda, Venezuela. Recuperado el 1 de Julio de 2020, de https://www.academia.edu/14876068/Funciona miento_y_aplicaciones_de_Celdas_de_Peltier

Martines L., E. L. (22 de Octubre de 2008). "Cálculo de la Temperatura de Punto de Rocío a Diferentes Valores de Presión". Santiago de Querétaro. Recuperado el 2 de Abril de 2019, de

https://www.cenam.mx/simposio2008/sm_2008 /memorias/M1/SM2008-M117-1098.pdf

MetAs \& Metrologos Asociados. (Noviembre de 2007). Temperatura de Punto de Rocio. MetAs \& Metrologos Asociados. Recuperado el 5 de Marzo de 2019, de http://www.metas.com.mx/guiametas/La-GuiaMetAs-07-11-punto-de-rocio.pdf

Patterson, G., \& Sobral, M. (2007). Efecto Peltier. Buenos Aires, Argentina: FCEyN, UBA.

Planet's Water Company. (s.f.). Recuperado el 10 de Noviembre de 2018, de Planet's Water Company: http://www.waterplanet.com/

Portillo, A. L. (02 de Junio de 2017). "Energía solar en México: su potencial y aprovechamiento". Recuperado el 15 de Mayo de 2019, de ciep.mx.

Rodriguez, J., \& Espinoza, E. (2017). La Industria Solar Fotovoltaica y Foto termica en Mexico. Ciudad de Mexico, Mexico: ProMéxico.

Sacristán, A. (2011). Obtención de agua apartir de humedad atmosférica empleando células de efecto Peltier. Madrid, España: Universidad Carlos III.

sde.mx. (2018). "¿Qué es un sistema fotovoltaico?". Recuperado el 24 de Marzo de 2019, de sde.mx: http://www.sde.mx/2018/08/10/que-es-unsistema-fotovoltaico/

Style, O. (2012). "Energia Solar Autonoma: Planificacion, Dimensionado e Instalacion de un Sistema Fotovoltaico Autonomo". Appropriate Technology. 
Tecnología, M. E. (s.f.). Mactronica: Electrónica y Tecnología. Recuperado el 15 de Mayo de 2019, de https://www.mactronica.com.co/sensor-delluvia-yl83-44083259xJM

Tornos, S., \& Sotelo, A. (Abril de 2006). Termoelectricidad, la energía del desequilibrio. Técnica Industrial 262. Recuperado el 2 de Marzo de 2019, de http://www.tecnicaindustrial.es/tiadmin/numero s/22/41/a41.pdf

Weather Spark. (2019). Weather Spark. Recuperado el 6 de Octubre de 2019, de https://es.weatherspark.com/y/2821/Climapromedio-en-Hermosillo-M\%C3\%A9xicodurante-todo-el-a\%C3\%B1o

ZeroMass Water. (s.f.). Recuperado el 12 de Noviembre de 2018, de ZeroMass Water: https://www.zeromasswater.com/ 